\title{
Towards Energy Efficient Shape Rolling: Roll Pass Optimal Design and Case Studies
}

\author{
Kan Huang ${ }^{1}$, Bin Huang ${ }^{1,2^{*}}$, Lei Fu and Kazem Abhary ${ }^{2}$
}

\begin{abstract}
Shape rolling is widely employed in the production of long workpieces with appropriate cross-section profiles for other industrial applications. In the development of shape rolling systems, roll pass design (RPD) plays an essential role on the quality control of products, service life of rolls, productivity of rolling systems, as well as energy consumption of rolling operations. This study attempts to establish a generic strategy based on hybrid modeling and an improved genetic algorithm, to support the optimizations of RPD and shape rolling operations at a systematic perspective. Objectives include improving the quality and efficiency of RPD, reducing energy consumption of shape rolling, as well as releasing the demands on costly trails and expert knowledge in RPD. Hybrid modeling based on cross-disciplinary knowledge is developed to overcome the limitations of isolated single-disciplinary models. And conventional genetic algorithm is improved for the implementation of optimal design. Targeting to integrate empirical data and published reliable solutions into optimizations, a parameters estimation method is proposed to transfer the initially misaligned models into a uniform pattern. A tool based on the Matlab platform is developed to demonstrate the optimal design operations, with case studies involved to validate the proposed methodology.
\end{abstract}

Keywords: Roll pass optimal design, Hybrid modelling, Genetic algorithm, Parameters estimation

\section{Introduction}

Shape rolling is a continuous high productive metalforming process, which enables the manufacturing of long workpieces with uniform cross-sections by applying plastic forces exerted through a set of grooved rolls $[1,2]$. As both the rolling energy consumption and the quality of rolled work-piece are heavily dependent on the section geometries of roll grooves as well as the rolling operating procedures, roll pass design (RPD), which aims to schedule the deformation operations and design profiles grooves of rolls, plays a critical role in shape rolling practices [3]. Therefore, the rolling energy consumption, quality of rolled products, cost efficiency, productivity, and service-life of rolls are directly affected by the RPD [4]. Literature review indicates that a typical RPD practice can be summarized as Figure 1. In the RPD implementations, three types of models are usually involved:

\footnotetext{
*Correspondence: bin.huang@unisa.edu.au

${ }^{1}$ School of Civil Engineering, Changsha University of Science and Technology, Changsha 410114, China

Full list of author information is available at the end of the article
}

processing models considering parameters such as number of passes required by the entire system, rolling schedule/sequences, rolling speed and temperature; physical models predicting the deformation of rolled materials and evaluating operational energy consumption; as well as the geometrical models defining the groove profiles for the dimension control of rolled workpieces $[5,6]$.

It is commonly accepted that the acceleration of industrialization has been boosting the global demand on more economical and sustainable rolling operations, which consequently drives the innovation and improvement of RPD $[4,7,8]$. However, due to the non-linear and highly uncertain deformation behaviors of rolled materials, complex rolling contexts, as well as the complicated interactions between thermal and mechanical phenomena, rolling practices are usually difficult to be numerically modelled, analyzed and predicted [9-11]. Thus, the exploration of efficient, accurate and flexible solutions for RPD becomes extremely attractive in the rolling system development and shape rolling operations.

Over the years, a great number of approaches have been developed to support RPD and optimization, which 


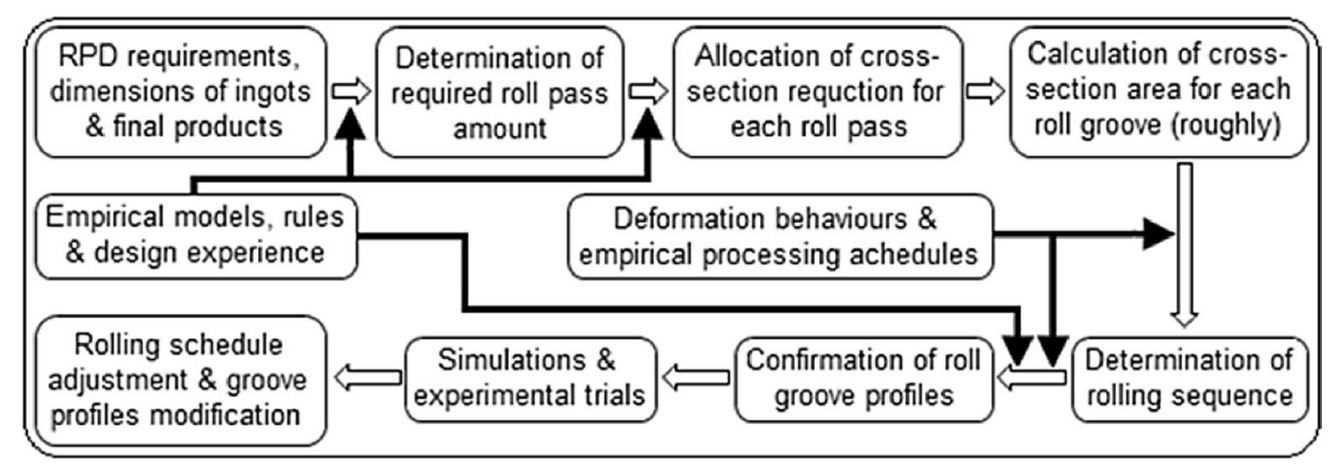

Figure 1 Schematic of a generic roll passes design procedure

can be basically classified into: 1) trial-and-error based empirical approaches; 2) finite element analysis (FEA) based simulation methods; and 3) artificial intelligence based expert systems. Since the rolling operations and RPD have been investigated for centuries, a large number of experimental models, approximate formulae and empirical rules were developed to guide the RPD. These approaches are popular for their ease of use [12]. However, due to the large number of approximation and assumptions applied in modeling and analysis, the scope and accuracy of these approaches are greatly limited [10]. In addition, empirical methods also suffer from the heavy dependence of expert knowledge, as well as costly and time-consuming trials, which thus affects the sustainability and stability of RPD [13]. The current development of computational science and FEA techniques has found a way to improve the modelling and analysis for roll pass optimal design (RPOD) [14, 15]. These methods can simulate rolling temperature evolutions, stress-strain states, as well as the complicated deformation behaviors with higher accuracy [16]. Although these FEA-based approaches are powerful in simulations, their large-scale application in RPOD is not yet efficient, due to the lower computational efficiency, the difficulty in deformation conditions setting, and the drawbacks in flexibility [17]. Recently, the development of intelligent design technology and expert systems has found a wide application in the optimal design of manufacturing systems and operations. Lambiase and Langella developed an expert system for the automated procedure of RPD [18]. While in a study of hot strip rolling, Bagheripoor and Bisadi introduced an artificial neural network (ANN) for the prediction of rolling force and torque [19]. These approaches possess significant strength in computeraided design using empirical data and rules, as well as design and operational experience integrated as expert knowledge [20-22]. Moreover, the flexibility and efficiency of RPD can also be enhanced with the engagement of those expert systems $[9,23]$. However, most expert systems developed in current studies have been primarily focused on single-pass optimal design or isolated rolling schedule optimizations, leaving the RPOD for the whole multi-pass rolling systems often neglected. This might be attributed to the lack of: 1) a universal model to describe the divers groove profiles in a numerical way; 2 ) a crossdisciplinary knowledge-based hybrid model to avoid benefiting one component at the cost of another one in optimization deliveries; and 3) an efficient and effective algorithm for the implementations of optimization.

This study endeavors to develop a generic strategy using cross-disciplinary knowledge-based hybrid modeling and an improved genetic algorithm (IGA) to support the RPOD at a systematic perspective. In order to conduct a clear and logical description for the proposed RPOD strategy, the rest of this paper is organized as follows. Section 2 discusses the theoretical background and framework. The formulation of hybrid model and objective functions is introduced in Section 3, followed by the improvements of genetic algorithm in Section 4. A case study implemented to demonstrate how the developed strategy works in Section 5, and finally Section 6 draws the conclusions and future works.

\section{Theoretical Background and Framework}

As mentioned in the previous section, to obtain a desired RPOD solution for the whole multi-pass rolling system, three distinct but inter-linked requirements need to be satisfied: pass amount determination, pass sequence identification and groove profiles definition. Therefore, the proposed RPOD is a three-dimensional optimization problem, with three types of parameters (processing, geometrical and physical/deformation) required to be processed simultaneously in the RPOD practices. The hybrid nature of this problem makes it difficult to obtain a desired solution using neither single-disciplinary knowledge nor isolated single-models. Therefore, a 


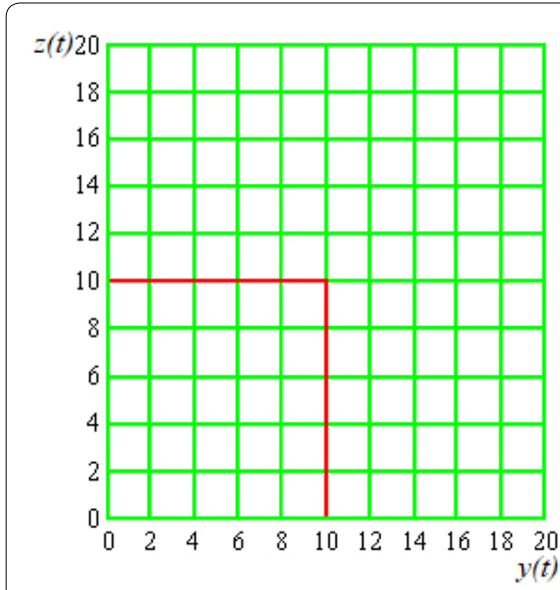

a A poly line with right angle

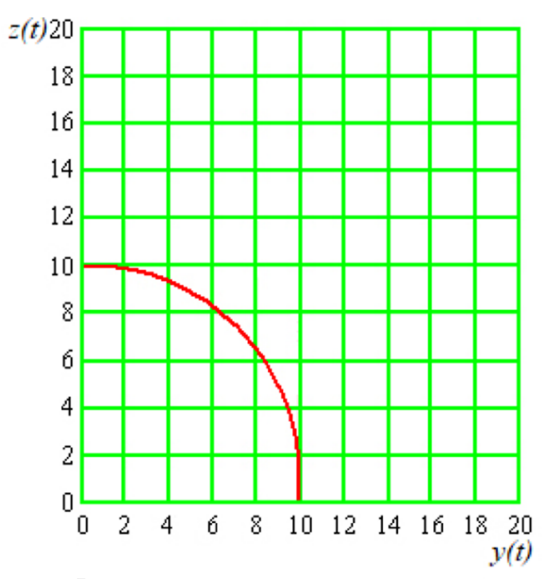

b An ideal circle with a diameter of 10

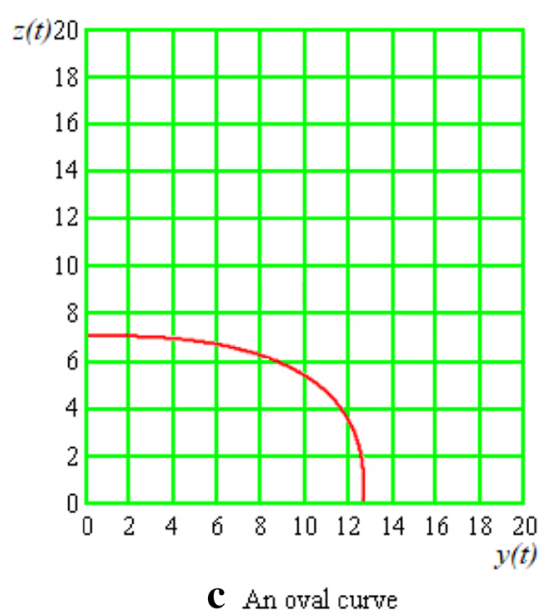

C An oval curve

Figure 2 A demonstration of the universal modelling (adopted from Ref. [3])

hybrid modelling strategy with cross-disciplinary knowledge is developed in this research.

RPOD for multi-pass rolling systems is a typical multiobjective-multi-constraint problem with the considerations of energy, design and cost efficiency, products quality and productivity, as well as the large number of design and process constraints imposed. Literature review indicates that GA is of great benefit to the handling of hybrid multi- objective-multi-constraint problems, due to its evolutionary nature, efficient parallel processing, independent on Pareto front, flexibility and ease to be conducted [22, 24, 25]. Thus, conventional GA is improved for the RPOD operations in this research. In addition, to integrate empirical geometrical data and solutions into the RPOD practices, a GA-based parameters estimation method is developed to realize the conversion of data format.

\subsection{A Universal Model for Geometrical Modelling}

In traditional approaches, predefined groove profiles are described numerically with diverse geometrical models and variables, therefore variables will be increased significantly with the number of passes applied, which consequently affects the solution representation and optimization handling. As shown in Eq. (1), suppose the cross-section profiles are located on the $y-z$ plane, a universal model was developed to describe all the groove profiles [26]. There are six parameters $(a, f, c, m, d$ and $g$ ) employed to modify the shape and curvature of the curve in this model. However, only five of them are independent variables, as $c$ is a dependent variable determined by $m$. For $m>0, c$ equals to 0 , otherwise the value of $c$ will be set as 1 . Figure 2 presents an example demonstrating the profile control with the five independent variables. Since the profiles are mirror symmetrical to both $y$ and $z$ axes, one quarter of these curves are presented.

$$
\left\{\begin{array}{l}
y(t)=\{(1-c) c\} \times\left\{\frac{a \cdot(\cos (t))^{p}}{f \cdot(\sin h(t))^{q}}\right\}, \\
z(t)=\{d-g\} \times\left\{\frac{\left(\sin \left(t^{u}\right)\right)^{p}}{f \cdot\left(\cosh \left(t^{u}\right)\right)^{q}}\right\} .
\end{array}\right.
$$

In this equation, $p=(1-c) \cdot e^{-f}, \quad q=c \cdot e^{-a}$, $u=2 \cdot e^{-m^{2}}$, and $t \in[0,2 \pi]$.

Since all the profiles employed in the multi-pass rolling system are constructed using the same model, both the rolling sequence and groove profiles can be determined by adjusting the values of independent variables. The geometrical modelling and rolling schedule planning can then be simplified.

\subsection{GA-Based Parameters Estimation}

Like other expert systems, design and processing data from successful RPD applications collected for the establishment of an empirical database to support the RPOD operations. Since geometrical models with diverse formulae were employed to define the groove profiles, data and solutions collected from other studies and applications cannot be used directly. Thus, a GA-based method is developed to convert data with different formats into the format required by the universal model.

According to the study conducted by Ref. [27], suppose two curves can be described by formulae $g_{1}(x)$ and $g_{2}(x)$, their similarity then can be assessed with the accumulative error $d\left(g_{1}, g_{2}\right)$ as expressed in Eq. (2):

$$
d\left(g_{1}, g_{2}\right)=\int_{c_{1}}^{c_{2}}\left|g_{1}(x)-g_{2}(x)\right| \mathrm{d} x .
$$




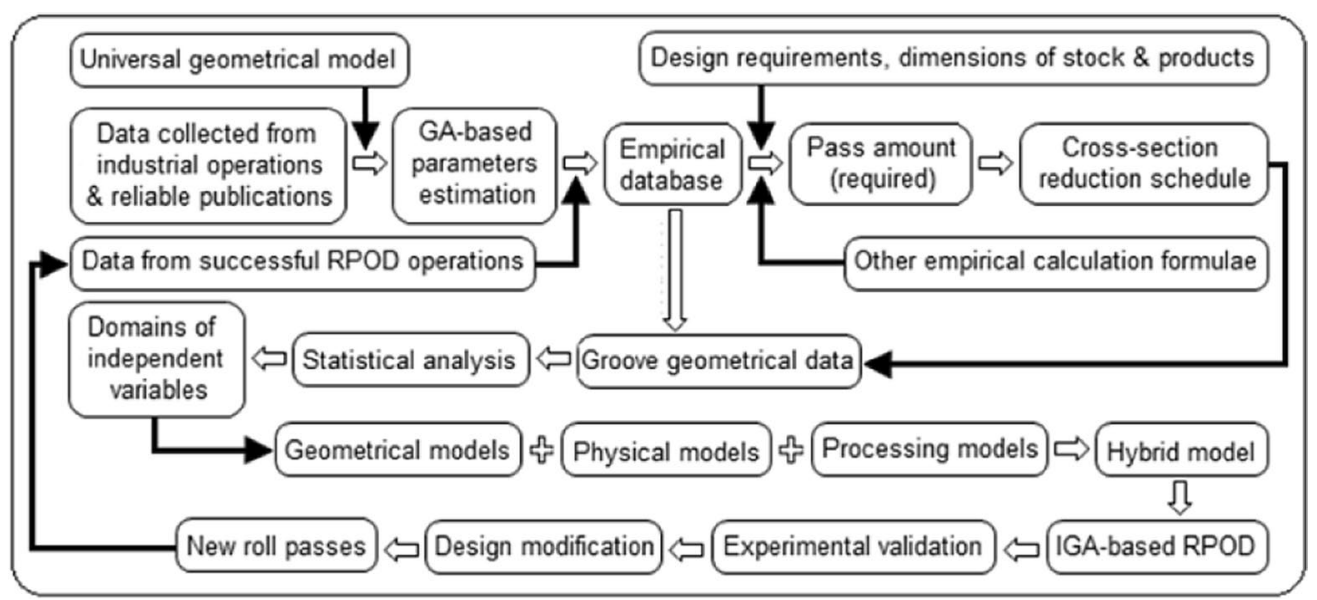

Figure 3 Architecture of the RPOD framework

Hereby, $\left[c_{1}, c_{2}\right]$ is the definitional domain for function $g_{1}(x)$ and function $g_{2}(x)$. With a given constant $\varepsilon$ (also defined as an acceptable error), if $d\left(g_{1}, g_{2}\right) \leq \varepsilon$, then the two curves are similar to each other, otherwise they are dissimilar. For some very complex scenarios, appraising the similarity of curves only through the accumulative errors might fail to satisfy the accuracy requirement. However, in this study, as the curves employed for the constructions of groove profiles are highly symmetric, similarity evaluation of profile curves with the accumulative error is acceptable. The objective function for the proposed GA-based parameters estimation then can be written as Eq. (3):

$$
f_{\text {est }}=\min \int_{c_{1}}^{c_{2}}\left|g_{1}(x)-g_{2}(x)\right| \mathrm{d} x .
$$

In this context, function $g_{1}(x)$ describes the profile curve based on the proposed universal model, while function $g_{2}(x)$ denotes the solution interpreted with the original model. A case study demonstrated in Ref. [26] indicates that this conversion method is effective. The accumulative error between original and rebuilt models is no more than $1.3 \%$, which is less than the acceptable $1.5 \%$ error in RPD.

\subsection{RPOD Framework}

This research endeavors to develop a generic approach to support the RPOD for the whole system. As shown in Figure 3, there are four phases for the RPOD operation. Phase 1 is proposed for empirical database establishment. Empirical data, rules and design models reflecting dimensions of grooves, operational parameters such as rolling temperature, force and torque, as well as required pass amount are collected from successful
RPOD implementations. After conversion by parameters estimation, an empirical database is built to support the RPOD operations. Phase 2 comes the identification of required pass amount and the domains of independent variables. In this research, the required number of passes is set as a variable, which allows to select the optimal one from a serial of feasible RPD solutions. The range of pass amount is identified by Eq. (4) based on the dimensions of stock and final products:

$$
n=\min \left\{n_{1,} n_{2}, n_{3}, n_{4,} n_{5}\right\}, \max \left\{n_{1}, n_{2}, n_{3}, n_{4,}, n_{5}\right\},
$$

where $n_{1}-n_{5}$ are the required numbers of roll passes for the entire rolling system obtained from diverse formulae $\left(n_{1}\right.$ is based on the reductions in height and width from ingot to final products, $n_{2}$ is calculated from the elongation coefficient, $n_{3}$ is identified by the height reduction ratio from ingot to final products, $n_{4}$ is based on the cross-section reduction ratio of each pass, and $n_{5}$ is obtained from empirical database) $[6,28] ;\lceil\cdot\rceil$ is a mathematical operator that finds the least integer which is greater than or equal to a real number within.

With the confirmation of pass amount, cross-section reduction ratios are then allocated followed by the determination of approximate area, height and width for each groove. Finally, domains of independent variables are determined by the rough dimensions, and the statistical analysis of empirical data.

Hybrid modeling and RPOD occur in Phase 3. Apart from the geometrical model, process and mechanical models are also integrated for hybrid modelling. Regression analysis and statistical techniques are employed to find out interactions among parameters, as well as to support the improvements of existing models [3]. Then the IGA-based RPOD is conducted to find out the optimal solution from the feasible domain. The final stage 
is designed for validation. Simulations and small-scale experiments are conducted to modify the RPOD solution for industrial applications.

\section{Hybrid Modelling and Constraints Handling}

\subsection{Hybrid Modelling}

The hybrid model proposed in this research is an integrated model which is based on cross-disciplinary knowledge. Design variables, such as geometrical, physical and processing parameters, are integrated in a hybrid matrix and considered simultaneously in the implementation of RPOD. This hybrid model focuses on both groove profiles as well as the processing characteristics of the whole rolling system, rather than the performance of RPD for any isolated single-pass.

In the process of optimization, to guide the GA searching and select the optimal solution from the feasible domain, objective functions have been established based on the RPD requirements. As this research is primarily focused on flexible, independent and effective RPOD operations, objectives were established as reductions of rolling energy demand and manufacturing errors, improvement of filling efficiency of grooves, and extension of rolls' service-life. The fitness function can then be described as

$$
\begin{array}{r}
f=\left[\omega_{1} \omega_{2} \omega_{3}\right] \times\left[f_{1}(x) f_{2}(x) f_{3}(x)\right]^{\mathrm{T}}, \\
\text { s.t. Constraints : } g_{i}(x) \leq 0 ; h(x)=0, \\
x \in X, i=1,2 .
\end{array}
$$

In this equation, $\omega_{1}, \omega_{2}$, and $\omega_{3}$ are weightings, which can be adjusted according to the design requirements. $f_{1}(x), f_{2}(x)$ and $f_{3}(x)$ are the objective functions established to minimum energy consumption and manufacturing errors, maximum service-life and improve filling efficiency of grooves. $g_{i}(x) \leq 0$ and $h(x)=0$ represent the inequality and equality constraints respectively, and $X$ is the domain including the lower and upper bounds of variables. According to the studies conducted by $\mathrm{Xu}$ et al. [6], for the $i$ th roll pass in the system, these objective functions can be described as:

$$
f_{1}(x)=\min \sum_{i=1}^{n} q_{i}=\min \left(\frac{\sum_{i=1}^{n} \alpha M_{Z_{i}} V_{i} \tau_{i}}{D_{k i}}\right) .
$$

Hereby, $q_{i}$ indicates the energy consumption. $M_{Z_{i}}$ refers to the rolling torque. $V_{i}$ represents the rolling velocity. $\tau_{i}$ demotes the rolling time, while $D_{k i}$ is the working diameter of rolls. Factor $\alpha$ indicates the constant coefficient, and $n$ represents the number of passes used in the multi-pass rolling system.

$$
f_{2}(x)=\min \sum_{i=1}^{i=n}\left(y_{i}^{2}+z_{i}^{2}-r^{2}\right) .
$$

$x_{i}, y_{i}, z_{i}$ are the coordinates of a given point located on the profile curve of the final pass, while $r$ denotes the ideal radius of final products.

The service-life of rolls can then be extended when a uniform wear of the roll groove is achieved, while the uniform wear of roll groove is subject to the uniform distribution of contact arc lengths and the value of contact area. In this research, $n_{1}$ points, which are uniformly distributed on the roll groove surface, are employed to control the uniform wear of roll groove for the maximization of roll service-life. Thus, the objective function for can be presented by

$$
f_{3}(x)=f_{4}(x)+f_{5}(x)
$$

$f_{4}(x)$ is the objective function for uniform distribution of contact arc lengths which can be presented as

$$
\begin{aligned}
f_{4}(x) & =\min \sum_{i=1}^{n_{1}}\left(y_{i}^{2}-\bar{L}^{2}\right) \\
& =\min \sum_{i=1}^{n_{1}}\left[\beta^{2}\left(R_{0}+h_{k} / 2-z_{i}\right)^{2}-\bar{L}^{2}\right] .
\end{aligned}
$$

In this equation, $\bar{L}$ is the mean value of contact arc lengths for those given points located on the roll groove profile curve. Parameters $y_{i}$ and $z_{i}$ indicate the coordinates of a given point on the profile curve respectively. $R_{0}$ is the minimum radius of rolls. $h_{k}$ refers to the height of the roll groove. $\beta$ represents the angle formed by the project plane and the vertical plane. $f_{5}(x)$ is another objective function for contact area optimisation which can be described as

$$
f_{5}(x)=\min \left(\frac{1}{\sqrt{A_{x}^{2}+A_{y}^{2}}}\right)=\max \left(\sqrt{A_{x}^{2}+A_{y}^{2}}\right),
$$

where $A_{x}$ and $A_{y}$ are the project areas of contact in the horizontal $x-y$ plane and the vertical $y-z$ plane respectively.

\subsection{Constraints Handling}

Constraint handling is a critical issue in optimizations. According to previous identification, there are four types of major constraints need to be satisfied: 1) permission angle of bite, which is an essential precondition for successfully bite of work-piece and rolling; and 2) tolerance requirement related to the dimension of final products; 
and 3) monotonicity requirement, sharp point avoidance and profile curve smoothness requirement, which benefit to the material flow in roll grooves, and consequently contribute to the quality of rolled work-pieces and rolls' service-life. The constraint from permissible bite angle $\alpha$ can be described as

$$
[\alpha]=\arccos \left(1-\Delta h / D_{k n}\right) \leq \alpha .
$$

In this equation, $[\alpha]$ is the bite angle used in a given pass, while $\Delta h$ indicates the height reduction of the bar. According to the study conducted by Beynon in Ref. [5], the working diameter $D_{k n}$ can be determined by

$$
D_{k n}=D-k_{4} h_{n} \text {. }
$$

Hereby, for square and diamond passes coefficient $k_{4}=1$; for oval grooves $k_{4}=0.819$; while for round and edging oval grooves $k_{4}=1.55 . h_{n}$ indicates the height of the $n$th roll groove.

It commonly accepted that broken lines are not suitable for the construction of groove profiles, since the inflection point (or sharp point) always results in stress concentration, which will affect rolling manufacturing as well as the wear and strength of rolls. In addition, outgoing bar with sharp edges (normally caused by sharp points on the groove-profiles) will lead to stress concentration and scratches of the following groove. Thus, the profile curve should be continuously derivable within the whole domain interval. In another word, for any point $\left(y_{0}, z_{0}\right)$ located on the profile curve, suppose $z=h(y)$ is the function employed to describe the relationship between the independent variable $y$ and the dependent variable $z$, then Eq. (13) need to be satisfied.

$$
\lim _{\mathrm{d} y \rightarrow 0} \frac{h\left(y_{0}+\mathrm{d} y\right)}{\mathrm{d} y}-h_{0}=0 .
$$

In the manufacturing process, tolerance control for final product is another crucial aspect for quality assessment. In multi-pass rolling operations, it is the final (or finishing) pass that plays the critical role for tolerance control. Thus, a model was established to guide the final pass optimal design. In rod rolling operations, for a given point $\left(x_{0}, y_{0}, z_{0}\right)$ located on the profile curve of the final pass, its dimension tolerance can be calculated as

$$
\Delta_{\left(x_{0}, y_{0}, z_{0}\right)}=\left|2 \sqrt{y_{0}^{2}+z_{0}^{2}}-r\right| \leq \varepsilon .
$$

In this equation, $r$ is the ideal radius of final products, while $\varepsilon$ represents the acceptable tolerance.

\section{Improvements of Genetic Operations}

As discussed in previous sections, the RPOD for the entire rolling system is a hybrid operation need to integrate rolling sequence scheduling with grove profiles

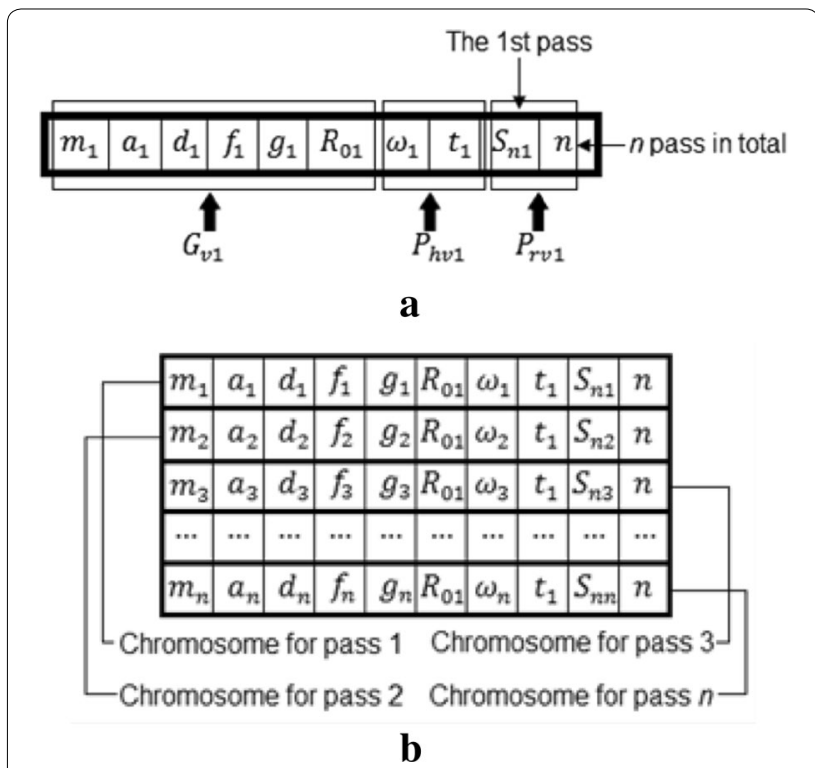

Figure 4 Chromosome representations for (a) a single pass and (b) a whole multi-pass system

design simultaneously. That makes conventional GA not suitable for the optimal design of a whole rolling system. Therefore, conventional GA is improved (hereafter IGA) by adding several application strategies for the RPOD operations developed in this research. A new solution representation is firstly developed for hybrid modelling, with the genetic operations improved in the following sections.

\subsection{A New Solution Representation for Optimization}

As identified in previous section, there are three types of variables employed in RPOD: geometrical $\left(G_{v}\right)$, physical $\left(P_{h v}\right)$ and processing $\left(P_{r v}\right)$. Thus, the generic form for a RPOD solution vector can be expressed as $X=\left\{G_{v}, P_{h v}, P_{r v}\right\}$. For an $n$-pass rolling system using the universal model for geometrical modelling, $G_{v}=\left\{m_{i}, a_{i}, d_{i}, f_{i}, g_{i}, R_{0 i}\right\}, \quad P_{h v}=\left\{\omega_{i}, t_{i}\right\}, \quad P_{r v}=\left\{S_{n i}, n\right\}$ (where $\omega_{i}$ is the angular velocity of rolls, $t_{i}$ is the initial temperature of stock, $S_{n i}$ is the sequence number, with $i=1,2,3, \ldots, n$, and $n$ is the total number of pass employed). To carry out the genetic operations, chromosome representation should be developed to encode the solution vector (or phenotype) into its genotype.

In this research, for a flexible and easier interpretation between the solution and the coding domains, grey-coding is employed. An individual solution for a single pass can be encoded as Figure 4(a). As a result, a solution for the whole multi-pass system can be expressed using a matrix as shown in Figure 4(b). 


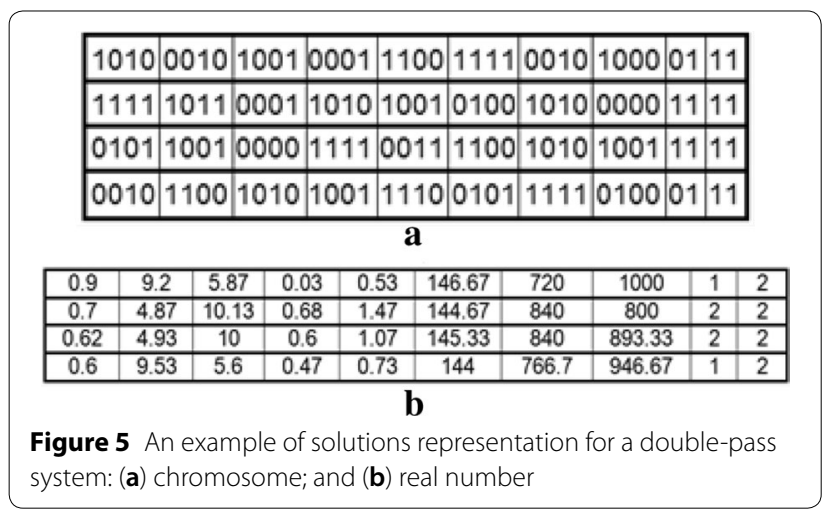

Since all chromosomes in the matrix have the consistent structure, they can share codes to simplify the encoding and decoding operations. However, different domains of variables are set for different roll passes according to the sequence number $S_{n i}$. In another word, variables in different chromosomes have the same format but different values, since different definition domains are applied in decoding. These definition domains are determined by the sequence number $S_{n i}$.

As shown in Figure 5(a), suppose there are four chromosomes in the design matrix (or design space) for a double-pass rolling system. Each variable is represented using a four bits grey-code. For the first pass, the definition domains for variables $m_{1}, a_{1}, d_{1}, f_{1}, g_{1}, R_{01}, \omega_{1}, t_{1}$ are $[0.5,1],[9,10],[4,6],[0,0.5],[0,1],[140,150],[700,800]$ and $[900,1000]$ respectively. For the second pass, the definition domains for variables $m_{2}, a_{2}, d_{2}, f_{2}, g_{2}, R_{01}, \omega_{2}, t_{2}$ are $[0.5,0.8],[4,5],[10,12],[0.2,0.8],[1,1.5],[140,150]$, $[800,850]$ and $[800,900]$. After decoding, the chromosomes can then be presented with real numbers.

\subsection{Operation of the IGA}

With the application of the proposed universal geometrical model, rolling sequence can be determined by adjusting the values of independent profile variables. Thus, the RPOD is shrunk into a two-dimensional optimisation problem. However, to obtain a global optimal solution from the feasible domain, fixed pass amount which is widely used in existing studies is replaced with a range. Consequently, one more computational circle is added to the normal GA operation flow (as shown in Figure 6).

\subsection{Fitness Evaluation and Calibration}

In the conduction of RPOD for the whole system, the chromosome population contains solutions for all passes. These solutions have the same chromosome representation but different domains for encoding and decoding.

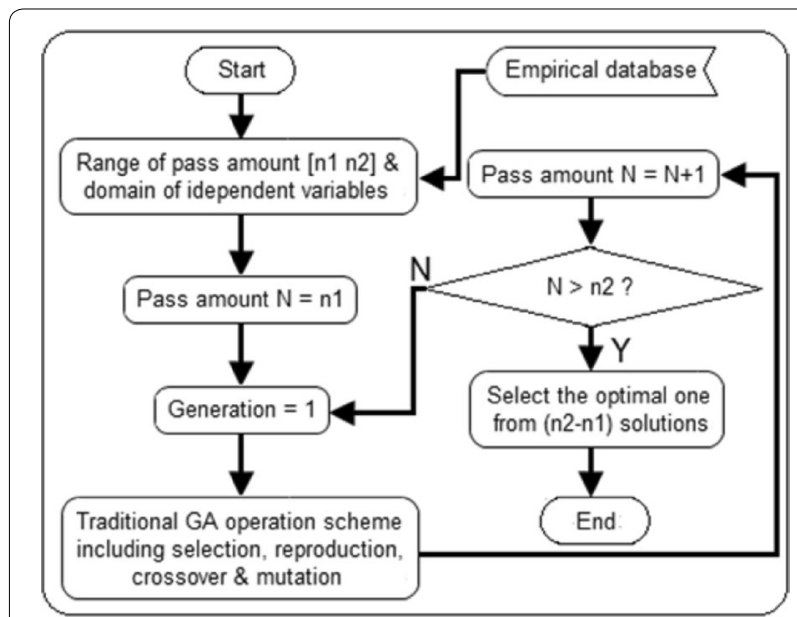

Figure 6 Flowchart of the IGA-based RPOD

Here, domain selection is determined by the sequence number (or identification number). A parallel decoding strategy, which can be expressed as Figure 7, is employed in this research to improve the operation efficiency.

To overcome the Hamming Cliff (which is a serious problem, appears in the crossover and mutation operations), grey-code is employed for coding operations. Therefore, the decoding procedure contains two steps: firstly, the grey-code string (which represents a chromosome or solution) is converted into a binary string through applying an $x$ - or operation bit-by-bit. The second step is to convert the binary string into real numbers according to the domains of variables.

Suppose the domain of a variable is $\left[U_{1} U_{2}\right]$ and the related binary string is $b_{n} b_{n-1} \ldots b_{1}$, then the value of this variable can be calculated from

$$
r_{n}=U_{1}+\left(\sum_{i=1}^{n} b_{i} \cdot 2^{i-1}\right) \cdot \frac{U_{2}-U_{1}}{2^{n}-1} .
$$

Since in some circumstance fitness values of chromosomes from the same population group might approximate to the mean value of the population fitness, which is unfavourable for the evolution and global searching, fitness calibration is required. In this study, a linear scaling calibration is developed, which can be expressed as

$$
F i t^{\prime}=\frac{F i t_{\mathrm{avg}}\left(F i t-F i t_{\mathrm{min}}\right)}{F i t_{\mathrm{avg}}-F i t_{\mathrm{min}}},
$$

where Fit is the original fitness value of a given chromosome, Fit ${ }^{\prime}$ is the new fitness value of this chromosome after calibration, Fit $t_{\text {avg }}$ is the average fitness of the current population, and $F i t_{\min }$ is the minimal fitness of the current population. 


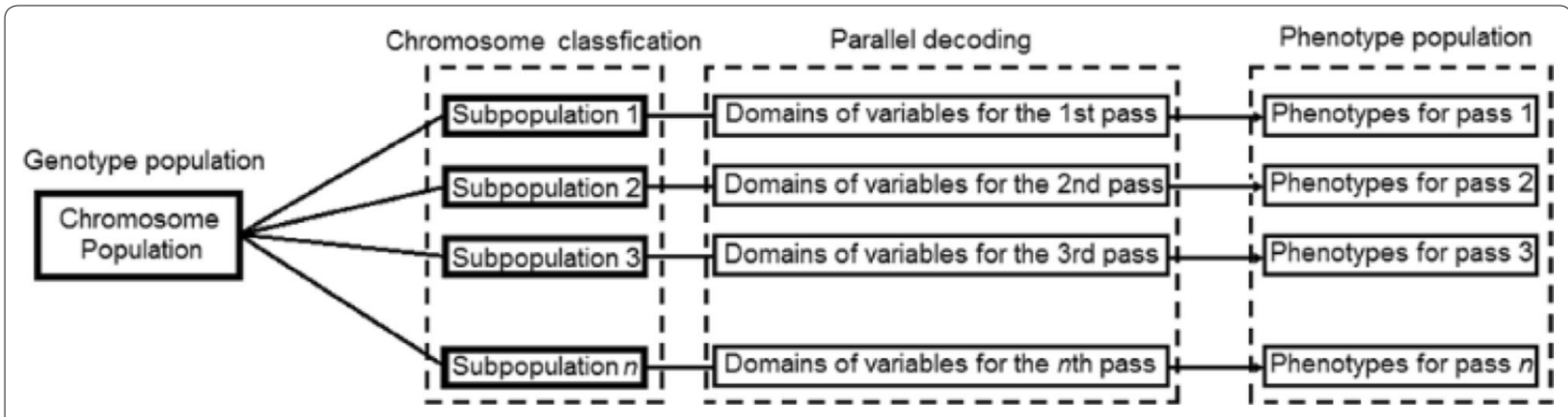

Figure 7 Scheme of parallel decoding

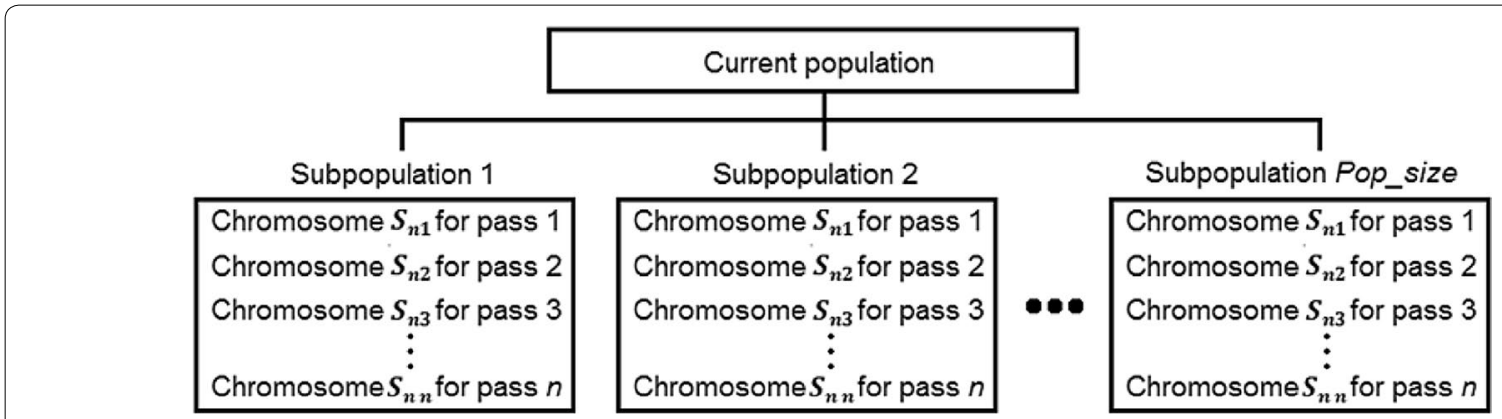

Figure 8 Scheme of population classification for selection operation

\subsection{Reproduction Operations for the IGA}

After the fitness evaluation and calibration for the initial population, the GA operations are executed until the specified termination conditions are satisfied or the maximum generation is reached. According to the features and mechanism of the IGA, there are three operations included in the IGA-based searching: selection, crossover and mutation.

\subsubsection{Selection Operation}

In GA, the selection operation is employed to select parents from current population to generate off-springs used in other genetic operations. In this research, the roulette wheel approach, which is widely adopted in most GA practices [29], is chosen for the selection procedure. To ensure the implementation of other genetic operations, chromosome classification is operated based on the sequence number of each chromosome. However, different from the chromosome classification carried out for parallel decoding, in this operation, chromosomes representation for different passes are assigned in the same subpopulation to ensure that each subpopulation represents a solution for the whole multi-pass rolling system (as shown in Figure 8).
After the reclassification of chromosomes, the fitness value for each subpopulation is computed, followed by the selection operation.

\subsubsection{Crossover Operation}

In the GA searching process, crossover, which is an important biological inspired operation, is widely employed to generate new populations. As previously discussed, the chromosome representation in this study is structured into a matrix form (as shown in Figure 4(b)). Thus, the uniform crossover which is a powerful and exploratory approach for the processing of matrix form crossover problems, is selected for the proposed RPOD method. The uniform crossover uses a fixed mixing ratio between two parents and evaluates each bit in the parent strings for exchange, can be described as follows.

Firstly, a crossover mask, which is a randomly generated binary matrix, is applied to determine the loci of genes for exchanges between parents. After that, offspring 1 will inherit the allele from parent 1 if the related locus of allele is 1 in the mask, otherwise it will inherit the allele from parent 2. Offspring 2 uses the opposite rule as offspring 1. For a five-pass rolling system, an example for such operation is shown in Figure 9. 


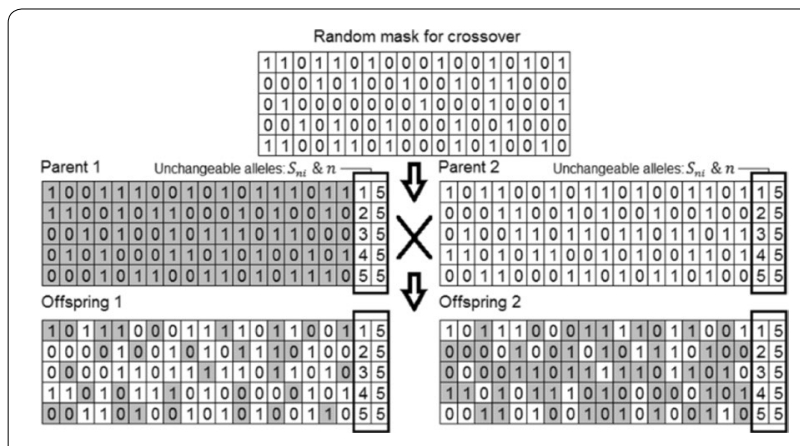

Figure 9 An example of uniform crossover in the RPOD

\subsubsection{Mutation Operation}

Another biological inspired genetic operator employed for GA searching is the mutation manipulation, which is designed to maintain the genetic diversity from one generation to the next through permutations of alleles. Essentially the mutation operation is a neighbour search technique. Current studies indicate that the bit-by-bit mutation is the most suitable operator for binary string representations, thus another random mask is generated for the mutation operation in this work. As with the crossover operation, the mutation manipulation is probabilistic and controlled by the mutation rate $p_{m}$. Thus, suppose each subpopulation has $\mathrm{n}$ chromosomes and every chromosome has $m$ bits of alleles, for each generation, the amount of alleles in current population to be mutated is $m \times n \times$ Pop_size $\times p_{m}$.

\section{Case Studies}

In this section, a case study is implemented to demonstrate the proposed RPOD strategy in this research. A Matlab program has been developed to support the demonstration and validation. In order to simplify the validation process, a case for 3-pass optimal design is employed. It is assumed that the ingot is an oval bar with the dimension of $32 \mathrm{~mm}$ in width, $16.5 \mathrm{~mm}$ in height, and $373.65 \mathrm{~mm}^{2}$ in area. The final product is a round bar with a diameter of $16 \mathrm{~mm}$. Work-piece material is 45 steels. Roll diameter is of $360 \mathrm{~mm}$, permission rolling force is $50 \mathrm{kN}$, permission rolling torque is $50 \mathrm{kN} \mathrm{m}$, power of drive motor is $570 \mathrm{~kW}$, and the speed range is 1000-1500 r/min.

The IGA parameters are set as follows: each variable is represented by a 10-bit grey code, thus the length of the chromosome is 90 bits with the population size of 100 and the generational gap of 0.9 . The crossover rate and mutation rate are 0.7 and 0.013 respectively. The simulation is terminated when the generation is over 100. As shown in Figure 10, there are three passes in the original solution extracted from the research conducted by Zhang in Ref. [30], with the pass sequence round-oval-round, and the manufacturing tolerance of $1.4 \%$.

Figure 11 indicates that after 50 iterations, the optimal solution is achieved. The geometrical parameters of passes obtained from RPOD are listed in Table 1, and technological parameters of original solution and optimal solution are listed in Table 2.

\section{Concluding Remarks and Future Work}

Comparative studies indicate that after optimization the total power consumption and rolling torque are decreased, while the working diameter of each pass is increased. By comparing with the original solution, it can be concluded that the dimensions of final products obtained from the optimal solution are closer to the desired dimensions. In addition, the cross-section reduction efficiency of intermediate passes (K3 and $\mathrm{K} 2$ ) is improved. However, that of the final pass is decreased, which actually is of benefit to the improvements of manufacturing precision and surface quality for the rolled work-pieces.

Based on the comparison made between the optimal solution obtained from IGA-based RPOD system and original solution from references, the following conclusions can be achieved:

- The dimensions of final product obtained from the RPOD are more desirable, thus the proposed IGA can support the RPOD of multi-pass systems with higher performance rolling.

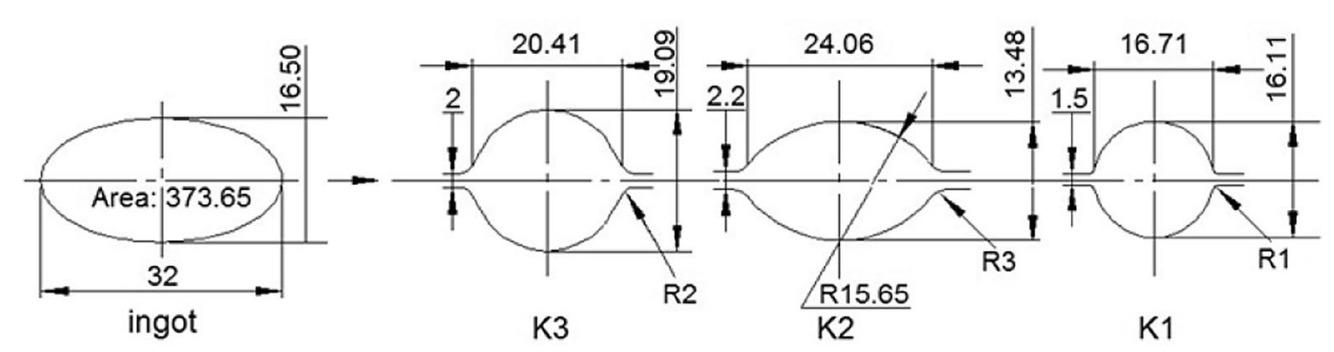

Figure 10 Solution from Zhang's [30] thesis 


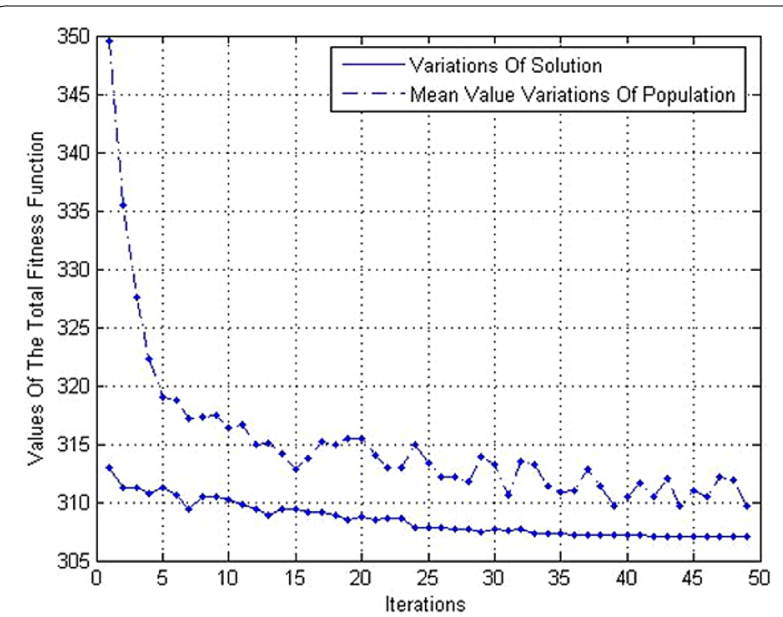

Figure 11 Performances trace for the optimal solution seeking

Table 1 Geometrical parameters of passes obtained from RPOD

\begin{tabular}{llll}
\hline Gen $=\mathbf{5 0}$ & K3 & K2 & K1 \\
\hline$m(\mathrm{~mm})$ & 0.83 & 0.83 & 0.83 \\
$a(\mathrm{~mm})$ & 10.00 & 13.47 & 8.10 \\
$f(\mathrm{~mm})$ & 0.16 & 0.17 & 0.17 \\
$g(\mathrm{~mm})$ & 0 & -1 & 0 \\
$d(\mathrm{~mm})$ & 9.85 & 6.21 & 8.05 \\
Roll gap $(\mathrm{mm})$ & 1.80 & 2.20 & 1.50 \\
Pass area $\left(\mathrm{mm}^{2}\right)$ & 299.50 & 233.84 & 201.96 \\
\hline
\end{tabular}

- The developed IGA-based RPOD approach can obtain an optimal solution for a three-pass rolling system in about three minutes, which indicates that the improved algorithm is efficient for RPOD.

- With the application of a new solution representation, the number of variables remains unchanged with the increase of pass amount, thus increase of pass number will not complicate the optimization.
- Figure 8 shows that the developed IGA possess high searching performance and fast convergence rate with the optimal solution achieved after only 50 iterations.

- Although only a simple 3-pass rolling system was employed for case study at the current stage, the proposed RPOD can be extended to the optimal design of more complex rolling system. Due to the proposed universal model and the new solution representation, the number of independent variables will not increase with the pass amount, nor the employment of passes with more complex groove profiles.

- Different from other RPOD expert systems, there is no predefined pass required in this approach, thus the proposed approach is more flexible and efficient.

- The proposed strategy is also suitable for other industrial problems which need to satisfy a simultaneously optimization for sequence planning and geometrical design.

Future work will focus on:

- Due to the time constraints, currently only a threepass rolling system is employed to validate the proposed strategy. Future works of this research will be extended to the validation of more complex rolling systems with more passes.

- Since the domains of independent variables employed for RPOD is determined by statistical analysis, more design data from successful solutions will be collected for the improvement of decision-making applied in the proposed approach.

- The proposed strategy is efficient and reliable. However, the industrial rolling operation is more complex with xmore passes. Therefore, in the following research further improvements will be proposed for the searching efficiency of the optimal algorithm. In addition, small-scale lab experiments will be carried out to study the deformation behavior and to further validate the developed methodology.

Table 2 Technological parameters of original and RPOD solutions

\begin{tabular}{|c|c|c|c|c|c|c|}
\hline \multirow{2}{*}{$\begin{array}{l}\text { Gen }=\mathbf{5 0} \\
\text { Reduction ratio }\end{array}$} & \multicolumn{3}{|c|}{ Original solutions } & \multicolumn{3}{|c|}{ Optimal solutions } \\
\hline & K3 & $\mathrm{K} 2$ & K1 & K3 & $\mathrm{K} 2$ & K1 \\
\hline & 1.21 & 1.20 & 1.21 & 1.25 & 1.28 & 1.16 \\
\hline Rolling speed (× $\left.10^{2} \mathrm{r} / \mathrm{min}\right)$ & 7.53 & 8.06 & 9.82 & 7.56 & 8.21 & 853.6 \\
\hline Rolling torque $(\mathrm{kN} \cdot \mathrm{m})$ & 5.03 & 4.51 & 3.24 & 4.66 & 4.35 & 3.18 \\
\hline Working diameter $\left(\times 10^{2} \mathrm{~mm}\right)$ & 3.40 & 3.46 & 3.44 & 3.45 & 3.50 & 3.48 \\
\hline Temperature $\left(\mathrm{k}^{\circ} \mathrm{C}\right)$ & 1.03 & 1.02 & 1.03 & 1.02 & 1.08 & 0.98 \\
\hline Power consumption ( $\times 10 \mathrm{~kJ}$ ) & 1.85 & 1.95 & 1.71 & 1.69 & 1.99 & 1.71 \\
\hline
\end{tabular}

Pass sequence is K3 to K2 (leading pass) to K1 (final pass), GA generations equal to 50 


\section{Authors' Contributions}

$\mathrm{KH}$ contributed to studies on the standards of construction elements rolling manufacturing. BH's role in this work is focused on hybrid modelling, algorithm development and programming. KA kindly provided supervision to this project. LF has been working with $\mathrm{BH}$ on algorithm design and experimental validations. All authors read and approved the final manuscript.

\section{Author Details}

${ }^{1}$ School of Civil Engineering, Changsha University of Science and Technology, Changsha 410114, China. ${ }^{2}$ School of Engineering, University of South Australia, Adelaide, South Australia 5095, Australia.

\section{Authors' Information}

Kan Huang, born in 1979, is currently working with the School of Civil Engineering, Changsha University of Science and Technology, China, as an Associate Professor and research degree supervisor. His research background including construction engineering, construction materials manufacturing, geological disaster control and handling. His current research is focused on optimal design and smart manufacturing of construction elements.

Bin Huang, born in 1984, is currently working with the School of Engineering, University of South Australia, Australia, as a Research Associate and Research Degree Supervisor, as well as working with Changsha University of Science and Technology, China, as a Guest Professor. Received his Ph.D. degree in Advanced Manufacturing and Mechanical Engineering from University of South Australia; Dr Huang' academic background is specialized in intelligent machinery and special robotics, intelligent design and optimization, as well as embodied control system development.

Lei Fu, born in 1997, is currently studying at School of Civil Engineering, Changsha University of Science and Technology, China, as High Degree Research student. Her research background and topic including construction engineering, sustainable manufacturing of construction materials, as well as green building technologies.

Kazem Abhary, currently working with the School of Engineering, University of South Australia, Australia, as a professor and research degree supervisor. Graduated from the University of Manchester, UK, 40 years ago in engineering, His research interests are focused on green (sustainable) mechanical and manufacturing, intelligent design for automation, as well as computer-aided design of mechanical systems. Prof. Kazem is currently involved in design for manufacture, as well as assembly and disassembly.

\section{Competing Interests}

The authors declare that they have no competing interests.

\section{Funding}

Supported by Scientific Research Foundation of Water Resources Department in Hunan Province of China (Grant No. CSCG-201808020002), Novelty in Civil Engineering of Key Discipline in Hunan Province of China (Grant No. 13ZDXK10), Research Study and Innovative Experiment of Undergraduates in 2018: Experimental Study on Grouting Model of Surrounding Rock of Tunne

Received: 13 June 2018 Accepted: 19 April 2019

Published online: 09 May 2019

\section{References}

[1] TTraub, X Chen, P Groche. Experimental and numerical investigation of the bending zone in roll forming. International Journal of Mechanical Sciences, 2017, 131-132: 956-970.

[2] S J Lee, S M Kim, D C Ko, et al. Design of roll profile in shape rolling of an irregular angle bar by the modified butterfly method. International Journal of Precision Engineering and Manufacturing, 2013, 14: 93-102.

[3] B Huang. Parameterized hybrid modelling for optimal roll pass design in hot rolling of light-round profiles. Adelaide: University of South Australia, 2013.

[4] LX Tang, J Y Liu, A Y Rong, et al. A review of planning and scheduling systems and methods for integrated steel production. European Journal of Operational Research, 2001, 133: 1-20.

[5] R E Beynon. Roll design and mill layout. Pittsburgh: Association of Iron and Steel Engineers, 1956
[6] CXu, Q SWang, C Zhang. Roll pass design for shape rolling. Beijing: Chemical Industry Press, 2009. (in Chinese)

[7] B Huang, KXing, K Abhary, et al. Development of energy-saving optimization for the oval-edging oval roll pass design using genetic algorithm. The International Journal of Advanced Manufacturing Technology, 2012, 61: 423-429.

[8] S A Hsiang, S L Lin. Modelling and optimization of caliber rolling process. Journal of Manufacturing Science and Engineering, 2007, 129: 77-83.

[9] F Lambiase. Optimization of shape rolling sequences by integrated artificial intelligent technique. The International Journal of Advanced Manufacturing Technology, 2013, 68: 443-452.

[10] $\vee$ Oduguwa, R Roy. A review of rolling system design optimization. International Journal of Machine Tools \& Manufacture, 2006, 46: 912-928.

[11] A Said, J G Lenard, A R Ragab, et al. The temperature, roll force and roll torque during hot bar rolling. Journal of Materials Processing Technology, 1999, 88: 147-153.

[12] S H Zhang, D W Zhao, C R Gao. The calculation of roll torque and roll separating force for broadside rolling by stream function method. International Journal of Mechanical Sciences, 2012, 57: 74-78.

[13] K Abrinia, A Fazlirad. Investigation of single pass shape rolling using an upper bound method. Journal of Materials Engineering and Performance, 2010, 19: 541-552.

[14] LW Zhang, CXYue, S Y Yuan, et al. Finite element simulation of rod and wire continuous rolling. Journal of Shanghai Jiaotong University (Science), 2011, 16: 195-198.

[15] R C Spoorenberg, H H Snijder, J C D Hoenderkamp. Finite element simulations of residual stresses in roller bent wide flange sections. Journal of Constructional Steel Research, 2011, 67: 39-50.

[16] U U Hanoglu, B Šarler. Multi-pass hot-rolling simulation using a meshless method. Computers \& Structures, 2018, 194: 1-14.

[17] E N Chumachenko, S A Aksenovl, V Logashina. Mathematical modelling and energy conservation for rolling in passes. Metallurgist, 2010, 54: 498-504.

[18] F Lambiase, A Langella. Automated procedure for roll pass design. Journal of Materials Engineering and Performance, 2009, 18: 263-272.

[19] M Bagheripoor, H Bisadi. Application of artificial neural networks for the prediction of roll force and roll torque in hot strip rolling process. Applied Mathematical Modelling, 2013, 37: 4593-4607.

[20] S H Kim, Y T Im. A knowledge-based expert system for roll pass and profile design for shape rolling of round and square bars. Journal of Materials Processing Technology, 1999, 89-90: 145-151.

[21] H C Kwon, Y T Im. Interactive computer-aided-design system for roll pass and profile design in bar rolling. Journal of Materials Processing Technology, 2002, 123: 399-405.

[22] M Poursina, N T Dehkordi, A Fattahi, et al. Application of genetic algorithms to optimization of rolling schedules based on damage mechanics. Simulation Modelling Practice and Theory, 2012, 22: 61-73

[23] B Huang, KXing, K Abhary, et al. Optimization of oval-round pass design using genetic algorithm. Journal of Robotics and Computer Integrated Manufacturing, 2012, 28: 493-499.

[24] A Abraham, L Jain. Evolutionary multiobjective optimization: Theoretical advances and applications. London: Springer, 2005.

[25] L H Randy, E H Sue. Practical genetic algorithms. 2nd ed. Hoboken: John Wiley and Sons, 2004.

[26] B Huang, KXing, K Huang, et al. Development of a geometric modelling strategy for roll pass optimal design. Robotics and Computer-Integrated Manufacturing, 2014, 30: 622-628.

[27] J Zhu, Z C Huang, X L Peng. Curve similarity judgment based on the discrete fréchet distance. Journal of Wuhan University (Nat. Sci. Ed), 2009, 55: 227-232.

[28] V N Djadechko. Roll pass design (Summary of lectures). Training Programme in The Iron and Steel Industry for The UN Fellowship Holders at Zaporozhstal Steel Works. (accessed on 8/09/2018).

[29] M Gen, R Cheng. Genetic algorithms and engineering design. New York: Wiley, 2000.

[30] N Zhang. The multi-objective optimal pass design based on GA. Qinhuangdao: Yanshan University, 2006. (in Chinese) 